\title{
YouTube and the single-rod contraceptive implant: a content analysis
}

\author{
Jennifer Paul, ${ }^{1,2}$ Christy M Boraas, ${ }^{2,3}$ Mildred Duvet, $^{2,4}$ Judy C Chang ${ }^{2,5,6}$
}

\begin{abstract}
${ }^{1}$ Kaiser Permanente, Greater Baltimore Medical Center, 7141 Security Boulevard, Baltimore, MD, USA

${ }^{2}$ Department of Obstetrics, Gynecology and Reproductive Sciences, University of Pittsburgh School of Medicine, Pittsburgh, PA, USA

${ }^{3}$ Department of Obstetrics, Gynecology and Women's Health, University of Minnesota Medical School, Minneapolis, MN, USA ${ }^{4}$ Department of Obstetrics and Gynecology, Leigh Valley Health Network, Allentown, PA, USA ${ }^{5}$ Magee-Womens Research Institute, Pittsburgh, PA, USA ${ }^{6}$ Center for Research in Health Care/Center for Women's Health Research and Innovation, University of Pittsburgh School of Medicine, Pittsburgh, PA, USA
\end{abstract} Dr Judy C Chang, Department of Obstetrics, Gynecology and Reproductive Sciences, MageeWomens Research Institute, 3380 Boulevard of the Allies, Pittsburgh, PA 15213, USA; jchang@mail.magee.edu

Received 1 August 2016 Revised 18 November 2016 Accepted 21 December 2016 Published Online First 20 January 2017

\section{Correspondence to}

\begin{abstract}
Background Since the internet has become an important source of contraceptive information with YouTube.com as the second most visited site, we analysed contraceptive implant YouTube videos for content and clinical accuracy.
\end{abstract}

Methods Using the terms 'contraceptive implant', 'Nexplanon' and 'Implanon', the top 20 results on YouTube by relevance and view count were identified. After excluding duplicates, single-rod implant videos in English were included. Videos were classified as providing a professional or patient perspective. Views, duration and comments were noted. Videos were rated for reliability, global quality scale and whether they were positive or negative about the implant. Inter-rater agreement was measured.

Results A total of 120 videos were retrieved; 52 were eligible for review. Less than $23 \%$ were professional videos; the majority reported patient experience ( $46 \%$ testimonials, $27 \%$ real-time procedure videos, $4 \%$ other). Patient videos had been posted a significantly longer duration of time than professional videos (364 vs 188 days, $p=0.02)$, were less reliable $(p \leq 0.0001)$ and were of lower global quality $(p<0.0001)$. Some $61 \%$ of implant testimonial videos were rated as 'positive experiences' and inter-rater agreement was very good $(\kappa=0.81)$. All testimonials mentioned side effects, commonly irregular bleeding and discomfort with insertion. A minority $(26 \%)$ reported misinformation.

Conclusions This study found that most of the information on YouTube pertaining to contraceptive implants is accurate, is presented from the patient's perspective, and promotes the method's use.

\section{CrossMark}

To cite: Paul J, Boraas CM, Duvet $\mathrm{M}$, et al. J Fam Plann Reprod Health Care 2017:43:195-200.

\section{INTRODUCTION}

Unintended pregnancy is a significant public health problem, representing nearly half of all pregnancies in the USA. ${ }^{1}$ Unintended pregnancy rates are

\section{Key message points}

- Many patients now use the internet and social media to disseminate and find health information.

- The majority of patient-created YouTube videos about contraceptive implants focused on patients' experience with the contraceptive implant and described positive experiences.

- Most patient-created YouTube videos on contraceptive implants contained accurate information.

particularly high among sexually active teenagers and women aged 20-24 years. ${ }^{1}$ Access to a range of contraceptive methods and the ability to use a desired method consistently and correctly has the potential to drastically reduce the rate of unintended pregnancy. Long-acting reversible contraceptive (LARC) methods, including the contraceptive implant, are highly effective. ${ }^{2}$ Use of these methods is increasing but overall is underutilised, especially among young women. ${ }^{34}$

Many factors influence a woman's contraceptive decision-making and choice. Clinician counselling can be helpful for patients, especially regarding ease of method use and effects on menstrual bleeding. ${ }^{5}{ }^{6}$ However, patients tend to seek information about contraception well in advance of initiating or switching methods. Patients were more likely to choose a method if they had positive perceptions about it prior to their clinic visit and had a friend or family member who was satisfied with her method, particularly when considering LARC. ${ }^{5}$

Women, especially adolescents, are increasingly using the internet and social 
media sites such as YouTube to access information about health and contraception. ${ }^{8}$ A recent report by the Pew Research Center showed that over $70 \%$ of people online use social media and users were more likely to be young women aged 18-29 years. ${ }^{9}$ Since its creation in 2005, the social media site YouTube. com allows users to upload videos clips on virtually any topic, many related to health. With over 100 million videos, YouTube has become an important resource for sharing personal testimonials about health and illness. ${ }^{10}$ Because of the exponential growth and popularity of YouTube and more users posting videos related to health, the website has potential for contraceptive education and to be a powerful influence in contraceptive choice, particularly with regard to LARC methods, which require fitting prior to initiation.

Studies have examined YouTube and health promotion as well as education about specific medical conditions. ${ }^{11-18}$ Empowering women with the information they need to make best use of available contraceptive methods will reduce unintended pregnancies. YouTube may represent an effective tool for disseminating information about contraception, including LARC. Exploration of intrauterine device (IUD) testimonials on YouTube suggests overall positive IUD experiences, despite reports of side effects and painful insertion. ${ }^{19}$ However, little is known about the type and quality of contraceptive implant information available on YouTube. The objective of our study was to characterise and analyse the content of information pertaining to the single-rod contraceptive implant available on Youtube.com.

\section{METHODS}

The University of Pittsburgh Institutional Review Board approved this study. On 26 February 2014, we searched the YouTube application programming interface to identify videos related to the contraceptive implant, using the search terms 'contraceptive implant', 'Nexplanon' and 'Implanon'. We prioritised the search for each term by view count and by relevance and collected the first 20 'hits' of results. Public health and information science literature supports the selection of the first 20 hits of a search. ${ }^{20-22}$ We excluded videos that were duplicates or that were primarily related to multi-rod contraceptive implants. We also excluded videos in a language other than English as our study team did not have the ability to perform content analysis on videos in other languages (Figure 1). For each video we collected basic information including title, uniform resource identifier (URL), date published, total number of views and comments, and number of 'likes' and 'dislikes'. We calculated the average number of views per day from the total number of views divided by the duration online to compare the groups.

Two independent researchers viewed the final sample of videos and a third researcher reviewed the video in the event of a discrepancy. Each video was classified based on source, either from a professional or patient perspective. We classified videos as 'professional' if posted by universities, professional organisations or individuals who identified themselves as trained healthcare personnel. We classified videos describing or depicting first-hand patient experience as 'patient' videos and then further classified them as either a testimonial or a real-time procedure video.

We evaluated each YouTube video for reliability using an established tool, the modified DISCERN. Singh et al. and Rittenberg et al. used a modified fivepoint DISCERN tool to assess rheumatoid arthritis and methotrexate injection videos on YouTube. ${ }^{23} 24$ Originally developed by Charnock and colleagues, the

\begin{tabular}{|l|}
\hline \multicolumn{2}{|c|}{ SEARCH TERMS } \\
1. "Nexplanon" \\
2. "Implanon \\
3. "Contraceptive Implant" \\
\\
SEARCH ALGORITHM \\
1. Relevance \\
2. View Count \\
\\
20 video hits per term per search algorithm \\
\hline
\end{tabular}

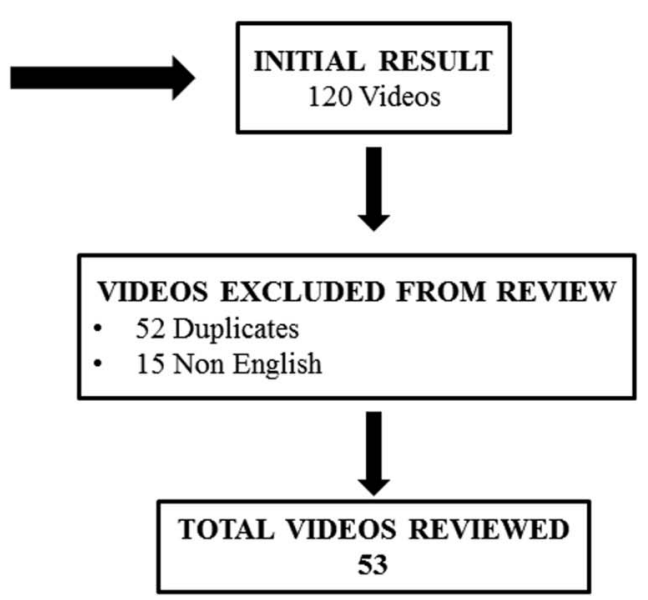

Figure 1 Video search and inclusion process. 
DISCERN tool enables evaluation of written health information. ${ }^{25}$ Components of the reliability assessment and global quality score are described in Box 1.

To assess reliability of contraceptive implant information in videos on YouTube, we allocated one point for each affirmative answer to the questions in the reliability assessment. The sum was noted as the video's reliability score. We also rated the quality of each video using the global quality scale. Bernard and colleagues originally published this scale and it has been used subsequently in other literature evaluating medical information on YouTube. ${ }^{23} 242627$

To capture patient experience, reviewers assessed each patient testimonial video for the presence or absence of information on five predetermined content domains: (1) reproductive health history (gravity, parity, unintended pregnancy), (2) contraceptive use history, (3) implant side effects, (4) implant access issues (cost, availability of providers) and (5) sources of implant information (medical professionals, social media). We rated each patient testimonial video as portraying an overall positive or negative experience with the contraceptive implant.

We performed all quantitative analyses using STATA V.12 (StataCorp LP, College Station, TX, USA). Descriptive statistics were used to describe the overall characteristics of the videos. We measured inter-rater agreement on the classification of patient experience

Box 1 Reliability assessment (A) and global quality scale (B) of YouTube contraceptive implant videos $^{2326}$

(A) Reliability: 1 point per question answered 'Yes'

1 Are the aims clear and achieved?

2 Are the reliable sources of information used? (i.e. publication cited, speaker is board certified)

3 Is the information presented balanced and unbiased?

4 Are additional sources of information listed for patient reference?

5 Are areas of uncertainty mentioned?

(B) Global quality scale

1 Poor quality, poor flow of the video, most information missing, not at all useful for patients

2 Generally poor quality and poor flow, some information listed but many important topics missing, of very limited use to patients

3 Moderate quality, suboptimal flow, some important information is adequately discussed but others poorly discussed, somewhat useful for patients

4 Good quality and generally good flow, most of the relevant information is listed, but some topics not covered, useful for patients

5 Excellent quality and excellent flow, very useful for patients (positive or negative) with the implant with Cohen's $\kappa$. We used Student's $t$-tests and Wilcoxon rank-sum tests where appropriate.

\section{RESULTS}

The initial YouTube search yielded 120 videos. Of these, we excluded 52 videos as duplicates and 15 as videos primarily in a language other than English. The remaining 53 videos were analysed (Figure 1). The total number of views for all included videos was 2221 118. Other characteristics of the total sample of videos are outlined in Table 1.

We classified all videos as either patient $(n=40$, $77 \%)$ or professional $(n=12,23 \%)$ videos. The majority of patient videos fell into two categories: a procedural video $(n=14,27 \%)$ depicting an actual insertion or removal procedure or a testimonial video $(n=24$, $46 \%)$ relating a woman's first-hand account of her experience with her contraceptive implant. The remaining two (4\%) patient videos showed neither a procedural nor a testimonial video and appeared to be created by individuals but discussed basic health information about the implant rather than personal experience. We excluded these two videos in the final analysis.

We compared the characteristics of the final sample of patient and professional videos and summarised the results in Table 2.

Patient videos were much less reliable and had lower global quality scale scores but longer duration on YouTube.

We focused the remainder of the analysis on patient testimonial videos, as these depicted women's opinions and perceptions of their implants. Overall, the women speaking in patient video testimonials reported positive experiences, although some videos $(n=5,22 \%)$ noted a mix of positive and negative feelings about the method. We rated the majority (61\%) of implant testimonial videos as 'positive experiences' and inter-rater agreement was very good (Cohen's $\kappa=0.81)$. Nine $(39 \%)$ videos expressed overall negative messages about the implant and a dissatisfied user

Table 1 Characteristics of contraceptive implant videos on YouTube

\begin{tabular}{ll}
\hline Characteristic & Data \\
\hline Number of videos & 52 \\
Number of views & $8902(4007,24232.5)$ \\
Number of views/day & $24.4(11,66.4)$ \\
Duration on YouTube since original posting (days) & $312.7(227.3)$ \\
Comments & $15.5(3,30)$ \\
'Likes' & $12(3,33)$ \\
'Dislikes' & $3(0.5,5.5)$ \\
\hline
\end{tabular}

Data are $n$ or median \pm (quartile 1, quartile 3 ) unless otherwise specified. ${ }^{*}$ Mean (SD). 
Table 2 Comparison of contraceptive implant videos stratified by source of video (patient or professional)

\begin{tabular}{lccc}
\hline Characteristic & Professional & Patient & $p$ \\
\hline Number of videos (\%) & $12(24)$ & $38(76)$ & \\
$\begin{array}{l}\text { Mean views/day } \\
\text { Mean duration on }\end{array}$ & $188.3(172.3)$ & $364.4(227.3)$ & 0.02 \\
$\begin{array}{l}\text { YouTube (days) } \\
\text { Mean number of }\end{array}$ & $1.5(0,42.5)$ & $19(11,31)$ & 0.062 \\
$\begin{array}{l}\text { comments } \\
\text { 'Likes' }\end{array}$ & $7(1,93)$ & $15(7,32)$ & 0.55 \\
'Dislikes' & $1(0,8)$ & $3(1,5)$ & 0.62 \\
Reliability & $3(3,4)$ & $2(1.5,2)$ & $<0.0001$ \\
$\begin{array}{l}\text { Global quality scale } \\
\text { score }\end{array}$ & $4.25(3.5,4.5)$ & $2.5(2,3)$ & $<0.0001$ \\
\hline
\end{tabular}

Data are median \pm (quartile 1, quartile 3 ) or $n$ (column \%) unless otherwise specified.

*Mean (SD).

experience. A minority of patient testimonial videos reported misinformation $(n=6,26 \%)$.

The content of patient testimonials varied, but users mentioned several aspects of implant use. Users most commonly addressed the implant side effects domain. All patient testimonial videos mentioned side effects. Most frequently, users mentioned an altered menstrual bleeding profile, including the majority $(n=14,61 \%)$ of videos reporting unpredictable bleeding and 35\% $(n=8)$ citing amenorrhoea. After changes in menstrual bleeding, users also mentioned pain with insertion. Thirty percent $(n=7)$ of videos cited significant pain during insertion, while $35 \%(n=8)$ reported relatively pain-free insertion. Seven $(30 \%)$ women mentioned a negative effect on their mood while using the method, while two (9\%) women noted that the device had no effect on mood. Six (26\%) women mentioned headaches, five (22\%) mentioned weight changes and four (17\%) mentioned breast symptoms. Four (17\%) women noted increased acne, with two (9\%) women reporting no change in acne. Other rarely mentioned side effects included device migration, decreased libido, insertion site haematoma, fatigue, insomnia, hunger, urinary frequency, or increase in premenstrual or early pregnancy-like symptoms. Misinformation reported in the videos was rare $(n=6,26 \%)$ but included mention of the fact that the device migrated 'deeper in the skin' over time and that it can cause liver damage or breast lumps. Users also frequently made the assumption in the video that their experience would be generalisable to all users.

Testimonial videos also mentioned experience with other contraceptives. Thirty-nine percent $(n=9)$ of testimonials noted use of other contraceptive methods prior to implant use, with oral contraceptive pills being the most frequently cited method. Other methods mentioned included natural family planning, the contraceptive ring, IUDs and condoms. We also collected data from testimonial videos regarding the poster's source(s) of information regarding the implant (friends/peers, internet sources, healthcare professional counselling). Over half $(n=13,54 \%)$ the patient testimonials discussed receiving counselling from a medical professional, although standard insertion practice includes informed consent explained by a medical professional prior to device insertion. Almost the same percentage $(n=12,52 \%)$ of testimonials mentioned they had gathered information from media sources including YouTube or other internet websites. Infrequently mentioned domains in patient testimonial videos included reproductive health history $(n=6)$ and any problems or issues related to obtaining contraceptive implants $(n=0)$.

\section{DISCUSSION}

Youtube.com is prolific and viewed by many users and healthcare consumers. Understanding content is important so that healthcare providers are aware of the potential information and messaging that may have influenced patients prior to their clinic visit. This study shows that most of the information on YouTube relating to contraceptive implants during the study period was from the patient perspective and promotes the use of implants. Additionally, most of the contraceptive implant information present on YouTube during the study was accurate.

Patient utilisation of the internet as a tool for education about healthcare topics is becoming increasingly prominent. Researchers have begun to characterise posted content in several fields to better understand what patients may encounter when doing internet searches, including specifically YouTube searches. ${ }^{11-18}$ Continued analysis of obstetrics and gynaecologyrelated content will help health professionals better understand what information and misinformation our patients may be encountering on the internet. As technology expands at an ever-increasing rate, in order to stay current the medical community needs to understand how patients interact with internet-related healthcare information, how they want to utilise this information, and how healthcare providers can harness social media and other novel means of information sharing in order to connect with and educate patients. Regarding our study findings, it is reassuring for healthcare providers that most of the information currently available about contraceptive implants on YouTube is accurate. The availability of patients' personal accounts of their experiences with the contraceptive implant can be a potentially more influential and useful mechanism for conveying information about the insertion process, side effects and benefits of the method.

This study, in common with other studies of usergenerated website content, is limited to an analysis of the content that was posted at one given time point (i.e. it is only a snapshot of the ever-changing body of information available on the internet). We attempted 
to minimise this limitation by sampling using two different methods (searching by relevance and view count) as well as employing multiple search terms. Our results may not necessarily be generalisable to other samples of YouTube videos, especially over time. The views expressed by the women in these videos cannot be assumed to represent the views of the entire female population, as women who post internet videos are a self-selected cohort. This group likely includes more frequent and savvy utilisers of technology. Also, our study did not include any analysis of the comments or postings related to any of the analysed YouTube videos. This is likely to be another rich and potentially influential source of opinions, experiences, and information or misinformation. Futures studies are needed to assess the content and accuracy of these comments.

Despite these limitations, our study findings have some important implications. As social media and other communication technology continue to expand and proliferate, healthcare providers need to consider using these communication modalities to augment their health education and counselling. Rather than discouraging use of the internet or social media for patient information-seeking, healthcare providers can acknowledge this as a potential resource, ask patients what they found and their thoughts about what they saw, and tailor their counselling to address patients' concerns and correct misinformation. Additionally, healthcare providers may wish to consider referring patients to specific videos or websites that contain reliable and accurate information, including YouTube videos, to reinforce their counselling messages. In one trial, patients who received contraceptive counselling augmented with written brochures were compared those whose counselling was augmented with a Facebook site with the same content information as the brochure. Contraception knowledge, satisfaction, and preferences for LARC methods were higher in the patients who received the Facebook information compared to those who received the written brochure. ${ }^{28}$ Potentially other social media sources such as YouTube can similarly enhance the effectiveness of providers' contraceptive counselling.

Future research should include attempting to understand how patients process and use the posted information when making contraception decisions. Additionally, most videos have many attached comments from both the video's poster as well as YouTube consumers. Analysing this content could yield a wealth of information about how these videos may be influencing other viewers. Our findings will hopefully prompt future research into harnessing social media, including YouTube.com, as a modality to help empower women in their contraceptive decision-making.

Competing interests None declared.

Provenance and peer review Not commissioned; externally peer reviewed.

\section{REFERENCES}

1 Finer LB, Zolna MR. Declines in unintended pregnancy in the United States, 2008-2011. N Engl J Med 2016;374:843-852.

2 Winner B, Peipert JF, Zhao Q, et al. Effectiveness of long-acting reversible contraception. $N$ Engl J Med 2012;366:1998-2007.

3 Whitaker AK, Sisco KM, Tomlinson AN, et al. Use of the intrauterine device among adolescent and young adult women in the United States from 2002 to 2010. J Adolesc Health 2013;53:401-406.

4 Kavanaugh ML, Jerman J, Finer LB. Changes in use of long-acting reversible contraceptive methods among U.S. women, 2009-2012. Obstet Gynecol 2015;126:917-927.

5 Egarter C, Frey Tirri B, Bitzer J, et al. Women's perceptions and reasons for choosing the pill, patch, or ring in the CHOICE study: a cross-sectional survey of contraceptive method selection after counseling. BMC Womens Health 2013;13:9.

6 Johnson S, Pion C, Jennings V. Current methods and attitudes of women towards contraception in Europe and America. Reprod Health 2013;10:7.

7 Schaefer M, Sheeder J, Teal S. Predictors of IUD initiation in adolescents. Contraception 2012;86:319. http://dx.doi.org/ 10.1016/j.contraception.2012.05.126

8 Sokkary N, Mansouri R, Yoost J, et al. A multicenter survey of contraceptive knowledge among adolescents in North America. J Pediatr Adolesc Gynecol 2013;26:274-276.

9 PewResearchCenter. The Demographics of Social Media Users - 2012. 2013. http://www.pewinternet.org/Reports/2013/ Social-media-users.aspx [accessed 18 November 2016].

10 Gabarron E, Fernandez-Luque L, Armayones M, et al. Identifying measures used for assessing quality of YouTube videos with patient health information: a review of current literature. Interact J Med Res 2013;2:e6.

11 Backinger CL, Pilsner AM, Augustson EM, et al. YouTube as a source of quitting smoking information. Tob Control 2011;20:119-122.

12 Chen HM, Hu ZK, Zheng XL, et al. Effectiveness of YouTube as a source of medical information on heart transplantation. Interact J Med Res 2013;2:e28.

13 Sood A, Sarangi S, Pandey A, et al. YouTube as a source of information on kidney stone disease. Urology 2011;77:558-562.

14 Ache KA, Wallace LS. Human papillomavirus vaccination coverage on YouTube. Am J Prev Med 2008;35:389-392.

15 Chou WY, Hunt Y, Folkers A, et al. Cancer survivorship in the age of YouTube and social media: a narrative analysis. J Med Internet Res 2011;13:e7.

16 Biggs TC, Bird JH, Harries PG, et al. YouTube as a source of information on rhinosinusitis: the good, the bad and the ugly. J Laryngol Otol 2013;127:749-754.

17 Babamiri K, Nassab RS. The availability and content analysis of melanoma information on YouTube. Plast Reconstr Surg 2010;126:51e-2e.

18 Keelan J, Pavri-Garcia V, Tomlinson G, et al. YouTube as a source of information on immunization: a content analysis. JAMA 2007;298:2482-2484.

19 Allen A, Nguyen B, Nagalla R, et al. Social media and the IUD - a YouTube content analysis. Contraception 2012;86:316. http://dx.doi.org/10.1016/j.contraception.2012.05.115

20 Madan AK, Frantzides CT, Pesce CE. The quality of information about laparoscopic bariatric surgery on the Internet. Surg Endosc 2003;17:685-687. 
21 Gordon JB, Barot LR, Fahey AL, et al. The Internet as a source of information on breast augmentation. Plast Reconstr Surg 2001;107:171-176.

22 Carroll MV, Shensa A, Primack BA. A comparison of cigaretteand hookah-related videos on YouTube. Tob Control 2013;22:319-323.

23 Singh AG, Singh S, Singh PP. YouTube for information on rheumatoid arthritis - a wakeup call? J Rheumatol 2012;39:899-903.

24 Rittberg R, Dissanayake T, Katz SJ. A qualitative analysis of methotrexate self-injection education videos on YouTube. Clin Rheumatol 2016;35: 1329-1333.
25 Charnock D, Shepperd S, Needham G, et al. DISCERN: an instrument for judging the quality of written consumer health information on treatment choices. J Epidemiol Community Health 1999;53:105-111.

26 Bernard A, Langille M, Hughes S, et al. A systematic review of patient inflammatory bowel disease information resources on the World Wide Web. Am J Gastroenterol 2007;102:2070-2077.

27 Delli K, Livas C, Vissink A, et al. Is YouTube useful as a source of information for Sjögren's syndrome? Oral Diseases 2016;22:196-201.

28 Kofinas JD, Varrey A, Sapra KJ, et al. Adjunctive social media for more effective contraceptive counseling: a randomized controlled trial. Obstet Gynecol. 2014;123:763-770. 